Research Article

\title{
Pharmacokinetics and Bioavailability Study of Tubeimoside I in ICR Mice by UPLC-MS/MS
}

\author{
Lianguo Chen, ${ }^{1}$ Qinghua Weng, ${ }^{1}$ Feifei Li, ${ }^{1}$ Jinlai Liu, ${ }^{1}$ Xueliang Zhang $\mathbb{D}^{1},{ }^{1}$ and \\ Yunfang Zhou iD ${ }^{2}$ \\ ${ }^{1}$ Wenzhou People's Hospital, The Third Clinical Institute Affiliated to Wenzhou Medical University, Wenzhou 325000, China \\ ${ }^{2}$ Laboratory of Clinical Pharmacy, The People's Hospital of Lishui, The Sixth Affiliated Hospital of Wenzhou Medical University, \\ Lishui 323000, China \\ Correspondence should be addressed to Xueliang Zhang; 854867181@qq.com and Yunfang Zhou; zyf2808@126.com
}

Lianguo Chen and Qinghua Weng equally contributed to this work.

Received 25 April 2018; Accepted 19 June 2018; Published 5 July 2018

Academic Editor: Josep Esteve-Romero

Copyright ( $\odot 2018$ Lianguo Chen et al. This is an open access article distributed under the Creative Commons Attribution License, which permits unrestricted use, distribution, and reproduction in any medium, provided the original work is properly cited.

\begin{abstract}
The aim of this study is to establish and validate a rapid, selective, and sensitive ultra-performance liquid chromatography-tandem mass spectrometry (UPLC-MS/MS) method to determine tubeimoside I (TBMS-I) in ICR (Institute of Cancer Research) mouse whole blood and its application in the pharmacokinetics and bioavailability study. The blood samples were precipitated by acetonitrile to extract the analytes. Chromatographic separation was performed on a UPLC BEH C18 column $(2.1 \mathrm{~mm} \times 50 \mathrm{~mm}$, $1.7 \mu \mathrm{m})$. The mobile phase consisted of water with $0.1 \%$ formic acid and methanol $(1: 1, \mathrm{v} / \mathrm{v})$ at a flow rate of $0.4 \mathrm{~mL} / \mathrm{min}$. The total eluting time was $4 \mathrm{~min}$. The TBMS-I and ardisiacrispin A (internal standard (IS)) were quantitatively detected by a tandem mass spectrometry equipped with an electrospray ionization (ESI) in a positive mode by multiple reaction monitoring (MRM). A validation of this method was in accordance with the US Food and Drug Administration (FDA) guidelines. The lower limit of quantification (LLOQ) of TBMS-I was $2 \mathrm{ng} / \mathrm{mL}$, and the calibration curve was linearly ranged from 2 to $2000 \mathrm{ng} / \mathrm{mL}\left(r^{2} \geq 0.995\right)$. The relative standard deviation (RSD) of interday precision and intraday precision was both lower than $15 \%$, and the accuracy was between $91.7 \%$ and $108.0 \%$. The average recovery was $>66.9 \%$, and the matrix effects were from $104.8 \%$ to $111.0 \%$. In this assay, a fast, highly sensitive, and reproducible quantitative method was developed and validated in mouse blood for the first time. The absolute availability of TBMS-I in the mouse was only $1 \%$, exhibiting a poor oral absorption.
\end{abstract}

\section{Introduction}

Tubeimoside I (TBMS-I), a triterpenoid saponin, is derived from the traditional Chinese bulb of Bolbostemma paniculatum (Maxim.). It was often used for the treatment of poisonous snake bite and inflammation [1]. In the past couple of years, additional attention was drawn to TBMS-I as it was reported to be a potential anticancer agent and appeared to be effective against several types of cancer, such as gliomas, breast cancer, colon cancer, and non-small cell lung cancer [2-5].

Pharmacokinetic studies are important in drug research and development which can provide systemic concentrations and exposure times of the drug for predicting a diverse range of efficacy- and toxicity-related events. To systematically examine the preclinical pharmacokinetic studies of TBMS-I in a reproducible and precise manner, a sensitive, fast, and validated analytical method for the determination of TBMS-I in biological fluids is imperative. However, the research on pharmacokinetics of TBMS-I lags behind compared to its pharmacological studies. Up to January of 2018, there was only one study report that published high-performance liquid chromatography coupled with tandem mass spectrometry (LC-MS/MS) for the determination of TBMS-I, which has been applied to the pharmacokinetic study in rats in 2007 [6]. But it had several drawbacks, such as long analysis time (more than $6 \mathrm{~min}$ ) and low sensitivity $(20 \mathrm{ng} / \mathrm{mL}$ ), especially 


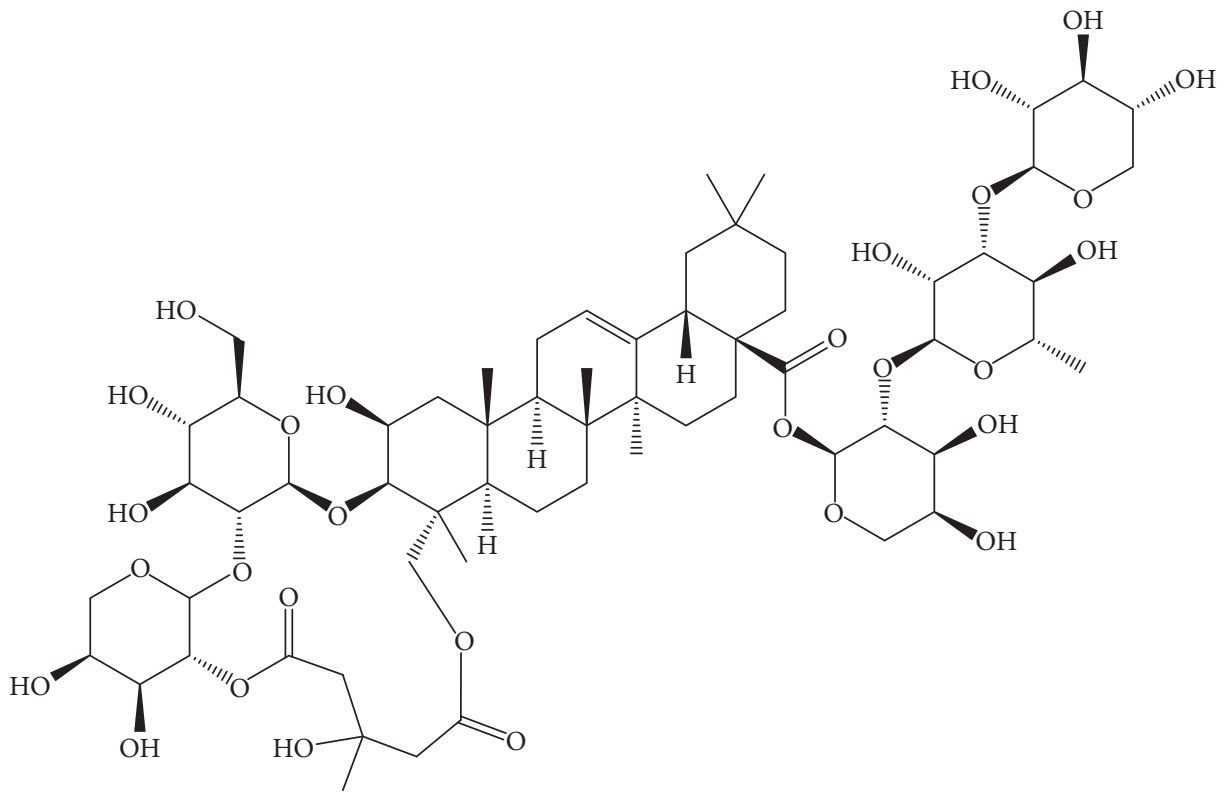

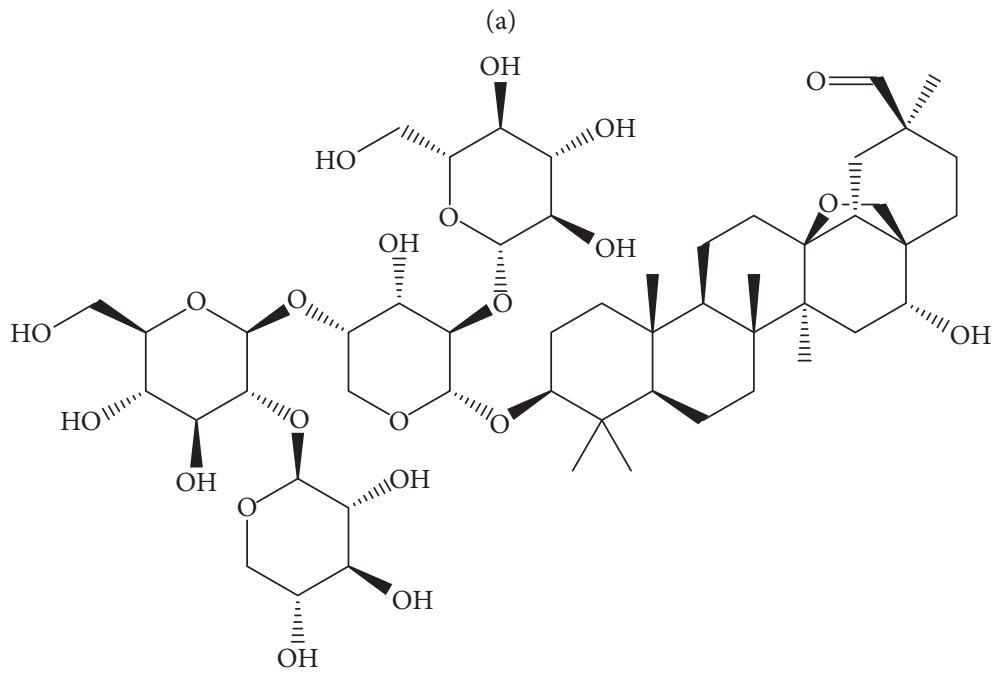

(b)

FIgURE 1: Chemical structures of tubeimoside I (a) and ardisiacrispin A (b).

requiring a large volume of plasma $(100 \mu \mathrm{L})$, which make it unsuitable for the serial blood sampling in mice pharmacokinetic evaluation [7]. In the last couple of years, the UPLC technique has attracted more and more concern along with the development of analysis techniques [8]. Compared with LC-MS/MS, the UPLC-MS/MS method was faster, more sensitive, and with higher sample throughput $[9,10]$. Meanwhile, its strong ability of isolating was more suitable for the analysis of the metabolism in vivo of the complex traditional Chinese medicine and complex compound [11, 12].

To the best of our knowledge, the profile of toxicity or pharmacokinetics of some drugs is alterable in different species [13-16], so it is not reasonable that these pharmacokinetic data are used directly in mice [17]. The mouse was chosen as the animal model to study the pharmacokinetics of TBMS-I in our study not only because of the reason mentioned above but also because the mouse is the most frequently used species for the preclinical efficacy $[18,19]$, toxicology
[20], biodistribution [21], and pharmacokinetic [22] studies to evaluate a potential anticancer agent, particularly with a limiting drug supply or specialized animal models in the early new drug discovery stage $[7,23]$.

Thus, we established a rapid, sensitive, and selective UPLCMS/MS method to quantitate the concentration of TBMS-I directly in the mouse utilizing low-volume whole blood after intravenous and oral administration in this study for the first time. A validation of this method was in accordance with the FDA guidelines. This method was successfully applied to the pharmacokinetics and bioavailability of TBMS-I in mice.

\section{Materials and Methods}

2.1. Experimental Materials. TBMS-I (purity >98\%; Figure 1(a)) and ardisiacrispin A (internal standard, purity $>98 \%$; Figure 1(b)) were purchased from Chengdu Mansite Biotechnology Co., Ltd. (Chengdu, China). HPLC-grade methanol 
and acetonitrile were bought from Merck (Darmstadt, Germany). HPLC-grade formic acid was supplied by Tedia (Ohio, USA). A Milli-Q system (Millipore, Bedford, USA) is used for generating ultrapure water. The ICR mice (male, weight 20-22 g, $n=12$ ) obtained were from the Laboratory Animal Center of Wenzhou Medical University (Wenzhou, China).

2.2. UPLC and Mass Spectrometric Conditions. The determination of analytes was carried out using the ACQUITY UPLC I-Class system equipped with a triple-quadrupole mass spectrometer (Waters Corp., Milford, MA, USA). MassLynx 4.1 software (Waters Corp.) was used to collect data and control the system.

TBMS-I and IS were separated on a UPLC BEH C18 column $(2.1 \mathrm{~mm} \times 50 \mathrm{~mm}, 1.7 \mu \mathrm{m})$ with a stable temperature of $40^{\circ} \mathrm{C}$. The mobile phases $\mathrm{A}$ and $\mathrm{B}$ were methanol and water with $0.1 \%$ formic acid, respectively. The details of gradient elution were as follows: the percentage of methanol was kept at $10 \%$ from 0 to $0.2 \mathrm{~min}$ and it reached $80 \%$ within $1.3 \mathrm{~min}$; then, it was kept at the same percentage for $0.5 \mathrm{~min}$ and subsequently it turned back to $10 \%$ for another $0.5 \mathrm{~min}$, and finally it was maintained at $10 \%$ for $2.5 \mathrm{~min}$. The flow rate was set at $0.4 \mathrm{~mL} / \mathrm{min}$, and the total elution time was $4.0 \mathrm{~min}$.

The mass spectrometer system for analysis was equipped with an electrospray source ionization (ESI) in a positive mode. The quantitative detection was performed in a multiple reaction monitoring mode at transitions $\mathrm{m} / z$ 1319.7 $\rightarrow 1187.6$ for TBMS-I (collision voltage $12 \mathrm{~V}$ and cone voltage $30 \mathrm{~V}$ ) and $\mathrm{m} / z 1083.5 \rightarrow 407.1$ for IS (collision voltage $72 \mathrm{~V}$ and cone voltage $100 \mathrm{~V}$ ). The capillary voltage was $2.3 \mathrm{kV}$. High-purity nitrogen as curtain gas and drying gas was set at $50 \mathrm{~L} / \mathrm{h}$ and $800 \mathrm{~L} / \mathrm{h}$, respectively. The temperature of the ion source and dissolvent was $150^{\circ} \mathrm{C}$ and $400^{\circ} \mathrm{C}$, respectively.

\subsection{Preparation of Stock Solutions, Quality Control (QC)} Samples, and Calibration Standards (CS). TBMS-I and IS were separately dissolved in methanol at a final concentration of $1.0 \mathrm{mg} / \mathrm{mL}$ as stock solutions. The working standard solutions were diluted from the stock solution using methanol. The standard working solution of IS was diluted with acetonitrile to the concentration of $50 \mathrm{ng} / \mathrm{mL}$. All solutions were stored at $4^{\circ} \mathrm{C}$ prior to analysis.

CS samples were prepared by diluting blank mouse blood into corresponding standard working solutions. A series of concentrations of standard solutions were prepared with TBMS-I stock solutions and serially diluted by using methanol. The final concentrations of TBMS-I were from 2 to $2000 \mathrm{ng} / \mathrm{mL}$, including $2,5,10,20,50,100,200,500,1000$, and $2000 \mathrm{ng} / \mathrm{mL}$.

Low-, mid-, and high-level QC samples of TBMS-I were similarly prepared at finial concentrations of 3, 190, and $1900 \mathrm{ng} / \mathrm{mL}$, respectively. All the solutions were stored at $-20^{\circ} \mathrm{C}$ until processed.

2.4. Sample Preparation. A $20 \mu \mathrm{L}$ aliquot of the mouse blood sample and $100 \mu \mathrm{L}$ of acetonitrile containing $50 \mathrm{ng} / \mathrm{mL}$ IS were added into $1.5 \mathrm{~mL}$ EP tubes [24]. After vortexing for
$1 \mathrm{~min}$, the specimens were centrifugated $(13000 \mathrm{rpm})$ for $10 \mathrm{~min}$ at $4^{\circ} \mathrm{C}$. Then, $80 \mu \mathrm{L}$ of the supernatant was collected; subsequently, a $2 \mu \mathrm{L}$ of the supernatant was injected into the UPLC-MS/MS system for analysis.

2.5. Method Validation. A validation of this method was in accordance with the FDA guidelines, including selectivity, linearity, precision, accuracy, recovery, matrix effect, and stability [25].

The chromatograms of blank mouse blood, blank blood spiked with TBMS-I and IS, and the real sample from mouse after dosing were used to estimate the selectivity of the UPLC-MS/MS method.

Calibration curves were generated by analyzing different concentrations of calibration samples on three consecutive days. The linear regressions of the peak area ratios $(y)$ of each TBMS-I to the corresponding IS versus the nominal concentration $(x)$ of TBMS-I were fitted over the range $2-2000 \mathrm{ng} / \mathrm{mL}$. Linearity was evaluated covering the concentration range 2-2000 ng/mL.

The interday precision, intraday precision, and accuracy were estimated by determining three concentrations of quality control samples $(n=6)$ on the same day and on three days in a row.

The recovery was calculated by comparison of the peak areas of TBMS-I and IS in the extracted low $(3 \mathrm{ng} / \mathrm{mL})$, middle $(190 \mathrm{ng} / \mathrm{mL})$, and high $(1900 \mathrm{ng} / \mathrm{mL})$ concentrations of QC samples with those of the extracted blank blood spiked with TBMS-I and IS at corresponding concentrations.

Matrix effects were tested by comparison of the peak areas of these new working solutions with those of the corresponding standard solutions diluted with methanol: $0.1 \%$ formic acid $(1: 1, \mathrm{v} / \mathrm{v})$ at equivalent concentrations, and this peak area ratio is defined as the matrix effect.

The stability of TBMS-I was tested under four conditions: storage in an autosampler at $4^{\circ} \mathrm{C}$, storage at room temperature for 2 hours, storage at $-20^{\circ} \mathrm{C}$ for a month, and three complete freeze-thaw cycles (from $-20^{\circ} \mathrm{C}$ to room temperature).

The stability of TBMS-I in mouse blood was obtained by comparing the areas of the newly configured QC samples with the corresponding three concentrations (3, 190, and $1900 \mathrm{ng} / \mathrm{mL}$ ) of standard samples.

2.6. Pharmacokinetic Study. Twelve mice were randomly and equally divided into two groups (A and B). Mice in the group A were injected sublingually with $5 \mathrm{mg} / \mathrm{kg}$ TBMS-I, and mice in the group B were given TBMS-I orally at a final concentration of $20 \mathrm{mg} / \mathrm{kg}$. The study protocol was approved by the Animal Care and Use Committee of Wenzhou Medical University. Mice were allowed to receive standard food and water ad libitum in a temperature-controlled room $\left(25^{\circ} \mathrm{C}\right)$ with a 12 -hour on and 12-hour off light cycle before the experiment.

Blood samples $(20 \mu \mathrm{L})$ were obtained from an individual mouse by tail vein bleeding in $1.5 \mathrm{~mL}$ tubes at 0 (prior to dosing), 0.0833, 0.5, 1, 1.5, 2, 3, 4, 8, 12, and $24 \mathrm{~h}$ after dosing. Six separate mice were used for sample collection and 


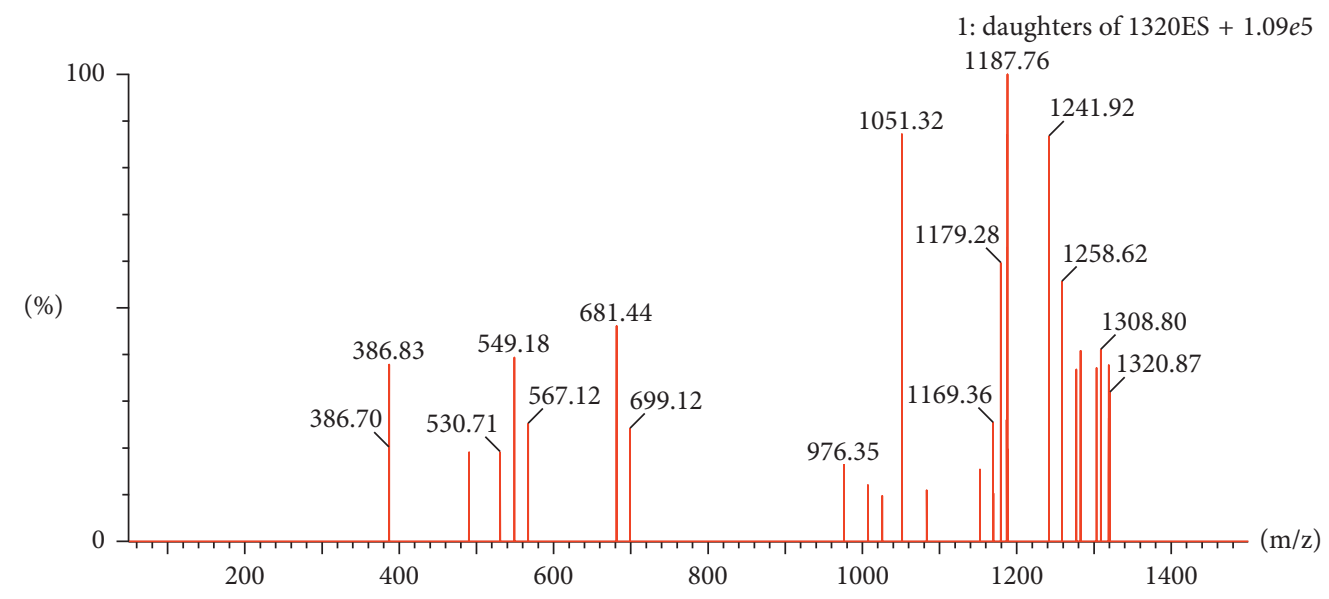

(a)

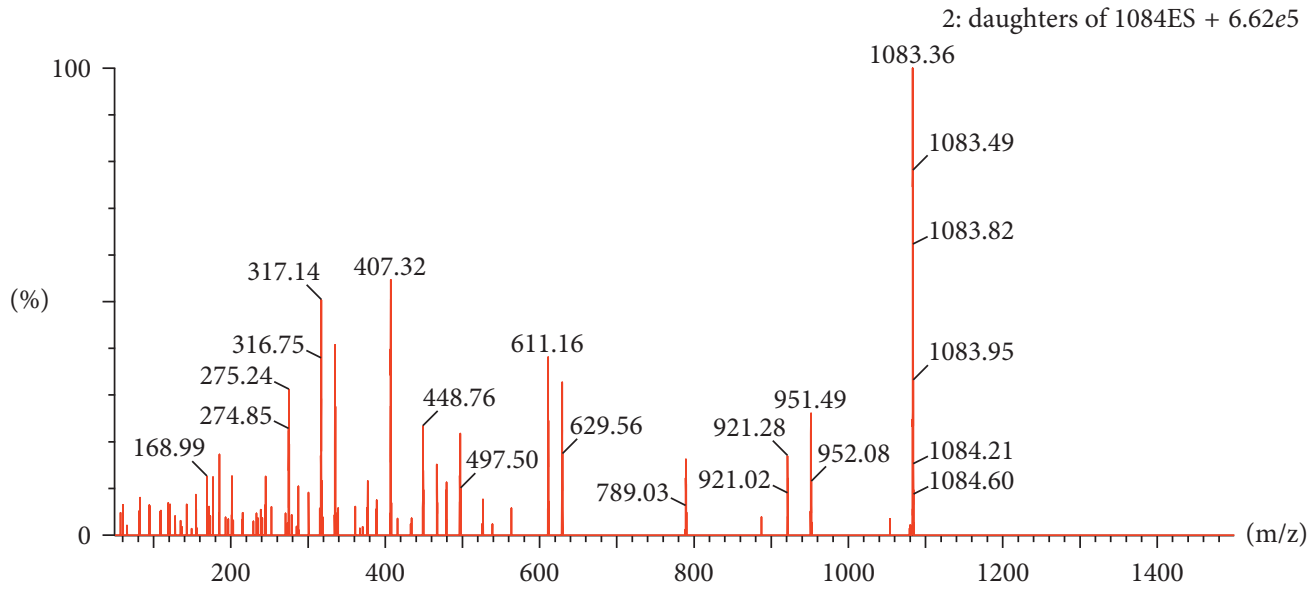

(b)

Figure 2: Mass spectrum of tubeimoside I (a) and ardisiacrispin A (b).

analysis at each time point. All the blood samples were directly stored at $-20^{\circ} \mathrm{C}$ until analysis. DSA 2.0 pharmacokinetic software (China Pharmaceutical University, China) was used to calculate the main pharmacokinetic parameters, including the area under the time-concentration curve (AUC), half-life $\left(t_{1 / 2}\right)$, the maximum of blood concentration $\left(C_{\max }\right)$, blood clearance rate $(\mathrm{CL})$, apparent volume of distribution $(V)$, and mean retention time (MRT). Bioavailability was calculated by absolute bioavailability $=100 \% \times \mathrm{AUC}_{\mathrm{po}} \cdot D_{\mathrm{iv}} /\left(\mathrm{AUC}_{\mathrm{iv}} \cdot D_{\mathrm{po}}\right)$, where $\mathrm{AUC}_{\mathrm{iv}}$ and $\mathrm{AUC}_{\mathrm{po}}$ are the $\mathrm{AUC}$ of the drug from $(0-\infty)$ after intravenous and oral administration, and $D_{\text {iv }}$ and $D_{\text {po }}$ are the single dosage of TBMS-I for the intravenous and oral administration, respectively.

\section{Results and Discussion}

3.1. Method Optimization. The mode of electronic source ionization (positive- or negative-ion mode) selection was often tested in a methodological study [26-30]. In this study, we chose the positive mode for the higher response achieved. According to the optimized results of mass spectrometric conditions, we can see that the daughter ions at $\mathrm{m} / z 1187.8$ and $m / z 407.3$ were the strongest and the most stable among abundant fragment ions produced, respectively, by TBMS-I and IS, which was presented in Figure 2. Thus, we selected $m / z 1319.7 \rightarrow 1187.8$ and $m / z 1083.4 \rightarrow 407.3$ for TBMS-I and IS, respectively.

In order to wash out the endogenous compounds as much as possible and avoid endogenous interference, the mobile phase was optimized [31, 32]. Several mobile phases were investigated on the ACQUITY BEH C18 column to obtain a perfect separation and a more symmetrical peak shape [33], including acetonitrile and water with $0.1 \%$ formic acid, acetonitrile and $10 \mathrm{mmol} / \mathrm{L}$ ammonium acetate solution $(0.1 \%$ formic acid), methanol and water $(0.1 \%$ formic acid), and methanol and $10 \mathrm{mmol} / \mathrm{L}$ ammonium acetate solution ( $0.1 \%$ formic acid). Among this, the mobile phase containing the mixture of methanol and water (including $0.1 \%$ formic acid) was chosen in this study for the best mass spectrometry peak and retention time using gradient eluting.

Proteins and other potential interference would affect the analysis of the mass spectrometry system [34, 35]. Therefore, an effective and simple sample preparation was a key point for establishing the UPLC-MS/MS method of TBMS-I. Liquid-liquid extraction (LLE) has the advantages 


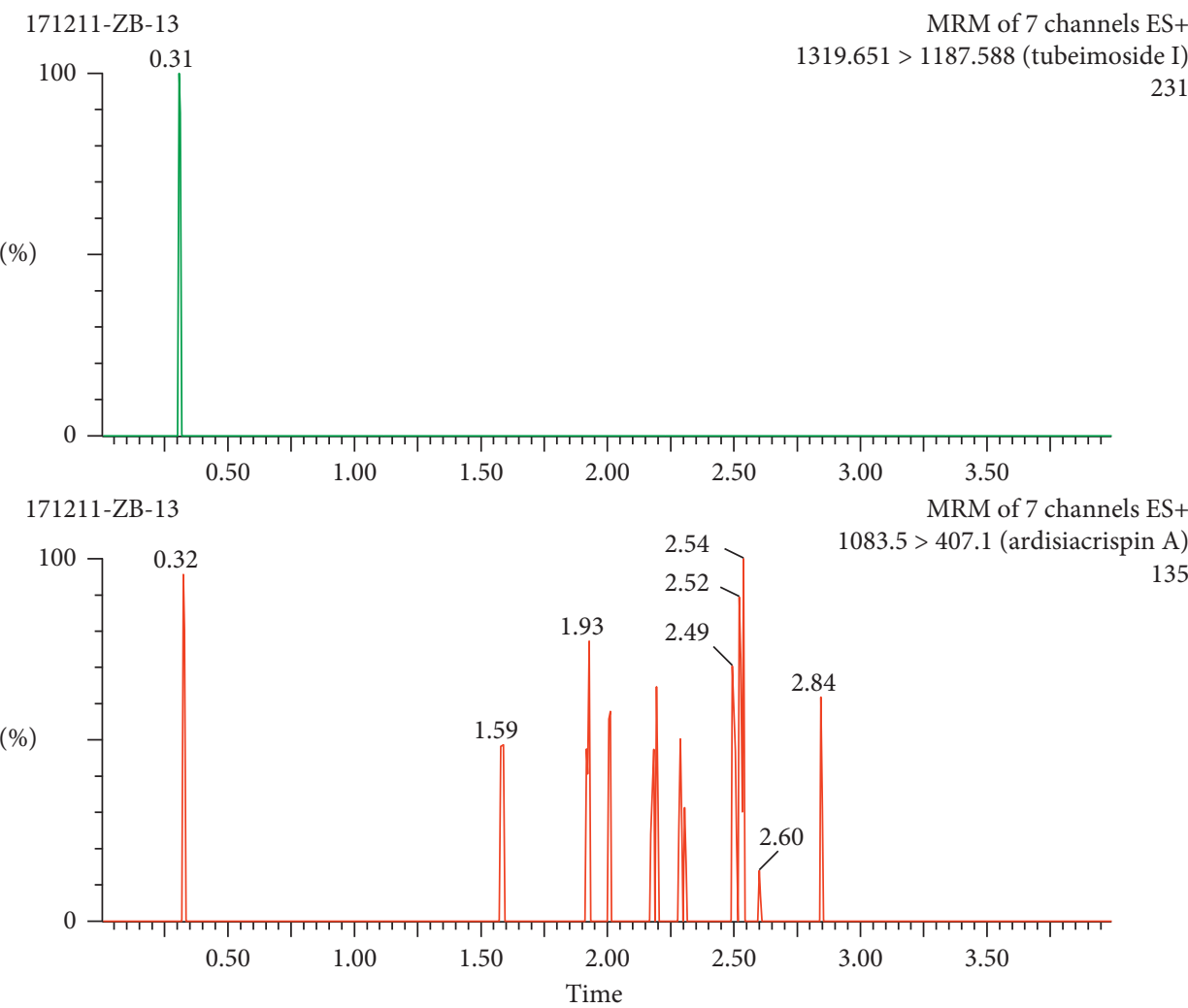

(a)

171211-ZB-11

MRM of 7 channels ES+

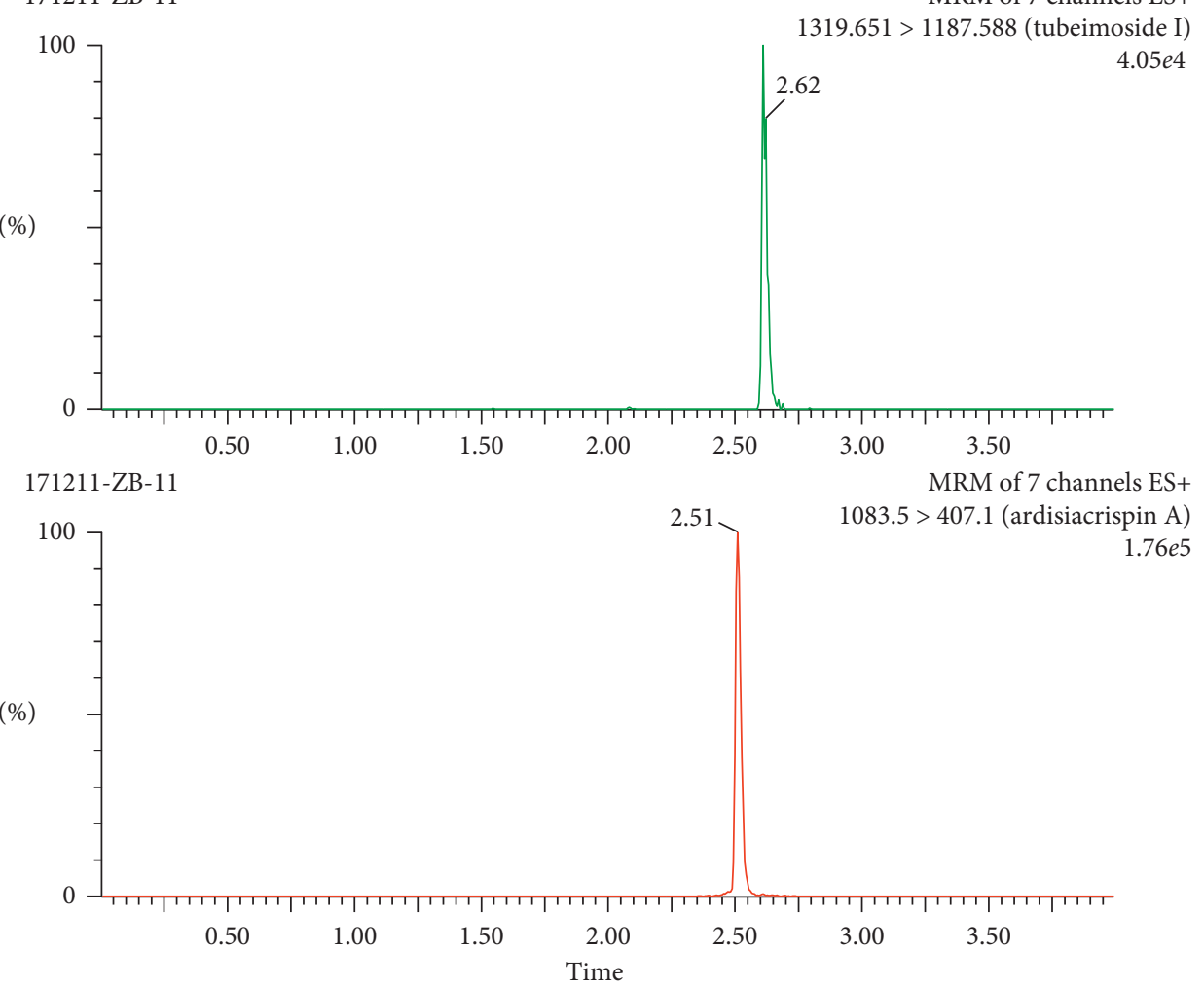

(b)

FIgURE 3: Continued. 


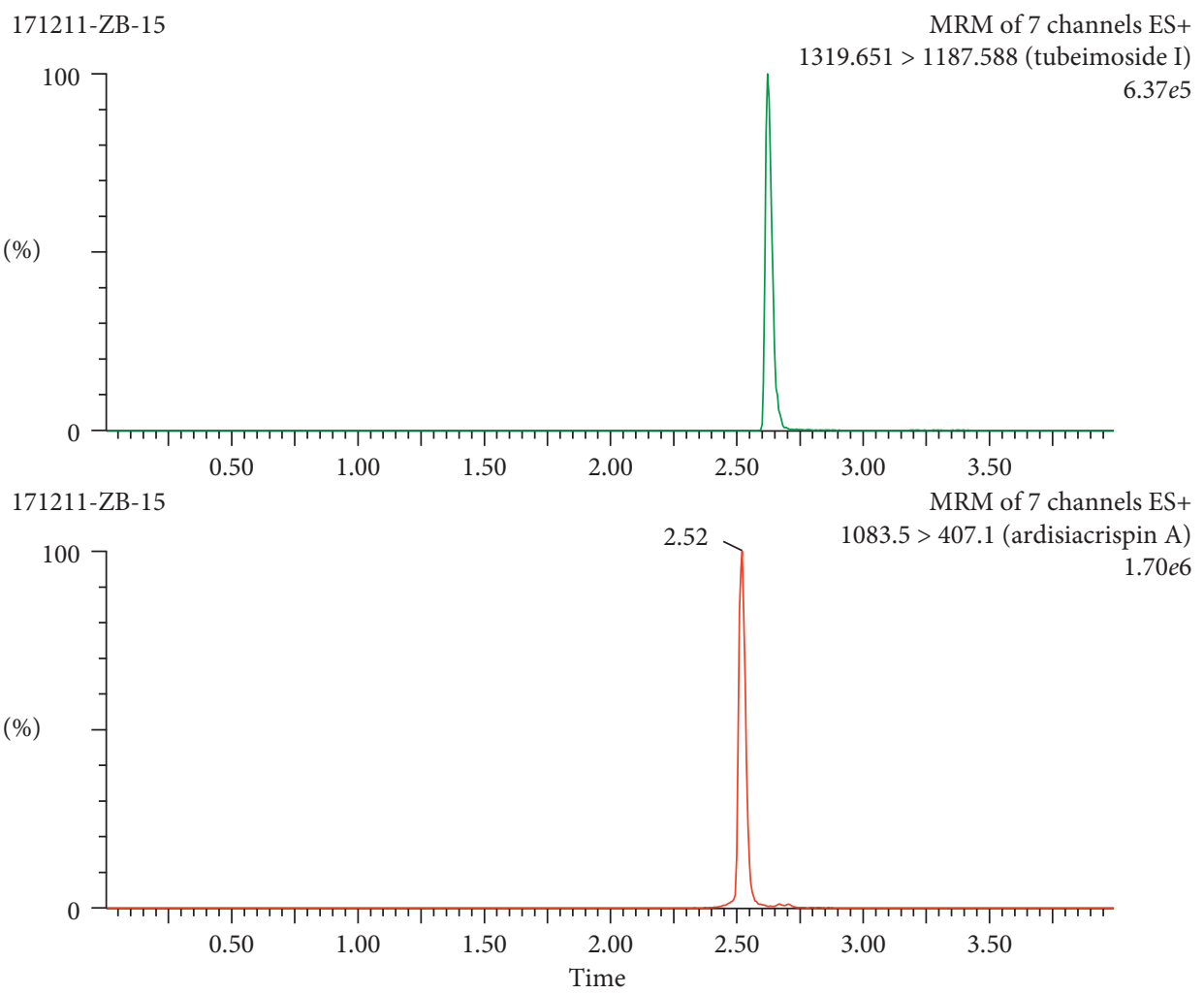

(c)

FIgURE 3: Spectrogram of the TBMS-I and IS: (a) a blank extract, (b) a blank extract with tubeimoside I and IS, and (c) the blood samples after administration spiked with IS.

of the high extraction rate and low limit of quantification [36], but this method required a long time for evaporation of the extracting solvent and large sample volumes. The number of blood samples, which can be taken from a mouse $(\sim 20 \mathrm{~g})$, is limited. Thus, it is not easy to get enough plasma after centrifuging for liquid-liquid extraction at each point (10 total time points in $24 \mathrm{~h}$ ) by tail vein transection bleeding. Taking these factors into consideration, only $20 \mu \mathrm{L}$ of blood samples was collected at different time points, and a one-step protein precipitation procedure for whole blood was chosen in our study following the example of the previous literature [37]. The small sample volume requirement further supports a serial blood sampling and enables entire pharmacokinetics from a single mouse which significantly reduces the numbers of mice used and inaccuracy of the pharmacokinetics because of individual differences $[7,23]$. Our method provides a simple, direct, and high-throughput assay for measuring TBMS-I because of the simple sample processing. The following precipitating solvents and their mixtures in different combinations and ratios were tested: methanol, acetonitrile, and acetonitrile-methanol. The results indicated that acetonitrile was a good precipitating reagent for the best recoveries for the analytes. Considering that blood samples are more complex than plasma, $20 \mu \mathrm{L}$ of the blood sample was mixed with 5 volumes of acetonitrile, which can not only provide higher recoveries and less matrix effect but also provide a sufficient supernatant volume for multiple injections for analysis. The level of TBMS-I in the supernatant obtained from the blood after protein precipitation and centrifugation is high enough to be detected by UPLC-MS/MS because the LLOD is $0.7 \mathrm{ng} / \mathrm{mL}$ and LLOQ is $2 \mathrm{ng} / \mathrm{mL}$ for TBMS-I, which will contribute to the assay of lower concentration of TBMS-I at the last time point for sample collection.

Internal standard was also an important task for establishing this method [38-40]. Tubeimoside I and ardisiacrispin A had a similar structure, so the retention time and the way of ionization of them are similar. In addition, ardisiacrispin A was a good choice for IS in our study because of its robustness, stability, absence of matrix effects, and reproducible extraction.

\subsection{Method Validation}

3.2.1. Selectivity. Figure 3 presents the ion chromatogram of a blank extract, a blank extract with TBMS-I and IS, and a blood sample from the caudal vein spiked with IS. The peaks of TBMS-I and IS appeared at 2.62 and $2.52 \mathrm{~min}$, respectively. No interfering peaks were found at or close by the retention times of TBMS-I and IS. The total runtime was 4.0 minutes.

3.2.2. Linearity. The regression equation of the calibration curve of TBMS-I was $y=0.00027776 x+0.0000866688$ ( $y$ represents the value of the peak area ratio of TBMS-I and IS and $x$ represents the concentrations of TBMS-I in blood). 
TABLE 1: Accuracy, precision, matrix effect, and recovery of the TBMS-I in mouse blood $(n=6)$.

\begin{tabular}{lcccccc}
\hline \multirow{2}{*}{ Concentration $(\mathrm{ng} / \mathrm{mL})$} & \multicolumn{2}{c}{ Precision (RSD \%) } & \multicolumn{2}{c}{ Accuracy $(\%)$} & \multirow{2}{*}{ Matrix effect (\%) } & \multirow{2}{*}{ Recovery (\%) } \\
& Intraday & Interday & Intraday & Interday & & \\
\hline 3 & 13.4 & 14.5 & 105.8 & 108.0 & 105.2 & 78.4 \\
190 & 11.2 & 10.6 & 94.4 & 91.7 & 104.8 & 68.6 \\
1900 & 5.3 & 8.4 & 101.3 & 104.1 & 111.0 & 66.9 \\
\hline
\end{tabular}

TABLE 2: The stability of TBMS-I under various storage conditions $(n=3)$.

\begin{tabular}{lcccccccc}
\hline & \multicolumn{2}{c}{$\begin{array}{c}\text { Autosampler } \\
\text { Concentration }(\mathrm{ng} / \mathrm{mL})\end{array}$} & \multicolumn{2}{c}{ Ambient $2 \mathrm{~h}$} & \multicolumn{2}{c}{$-20^{\circ} \mathrm{C} 30 \mathrm{~d}$} & \multicolumn{2}{c}{ Freeze-thaw } \\
& Accuracy & RSD & Accuracy & RSD & Accuracy & RSD & Accuracy & RSD \\
\hline 3 & 96.0 & 3.8 & 107.5 & 5.1 & 92.6 & 14.2 & 113.5 \\
190 & 106.2 & 6.7 & 108.0 & 4.8 & 109.9 & 9.4 & 111.0 & 11.2 \\
1900 & 104.1 & 5.5 & 95.8 & 4.4 & 91.7 & 6.0 & 90.3 & 7.5 \\
\hline
\end{tabular}

The correlation coefficient $r^{2}$ was 0.9976, which showed a good linearity. The LLOQ was $2 \mathrm{ng} / \mathrm{mL}$ with the signal-tonoise ratio ( $\mathrm{S} / \mathrm{N}$ ratio) of 10 for the determination of TBMS-I in mouse blood, and the lower limit of detection was $0.7 \mathrm{ng} / \mathrm{mL}$ with the $\mathrm{S} / \mathrm{N}$ ratio of 3 .

3.2.3. Precision, Accuracy, Recovery, and Matrix Effect. Table 1 shows the results of the precision, accuracy, recovery, and matrix effect. The RSD of interday precision and intraday precision was no more than $14 \%$ and $15 \%$, respectively. The accuracy was in the range of $91.7 \%$ to $108.0 \%$ at each QC level. All of the recoveries were above $66.9 \%$, and matrix effects were between $104.8 \%$ and $111.0 \%$. These data suggest that this method was satisfied with the pharmacokinetic study of TBMS-I.

3.2.4. Stability. The blood samples under the different storage conditions mentioned above $(n=3)$ were carried out the stability experiment (results shown in Table 2). In this study, the variations of each condition were within $14 \%$ and RSD was under $15 \%$, which indicated a reliable stability behavior of TBMS-I under the different storage conditions.

3.3. Pharmacokinetic Studies. Time-concentration curve of TBMS-I after oral and intravenous administration is shown in Figure 4. The pharmacokinetic parameters were calculated according to the noncompartment model (results are presented in Table 3 ). The $t_{1 / 2 z}$ was $2.3 \pm 0.5 \mathrm{~h}$ for oral administration and $6.8 \pm 5.6 \mathrm{~h}$ for intravenous administration, respectively. The $T_{\max }$ was $1.8 \pm 1.3 \mathrm{~h}$ after oral administration. The absolute availability was only $1.0 \%$. These results in mice were similar to that of rats described by Liang et al. [6].

\section{Conclusions}

A novel UPLC-MS/MS method for the quantitative measurement of TBMS-I in mouse blood has been developed and validated. The application of this method for the

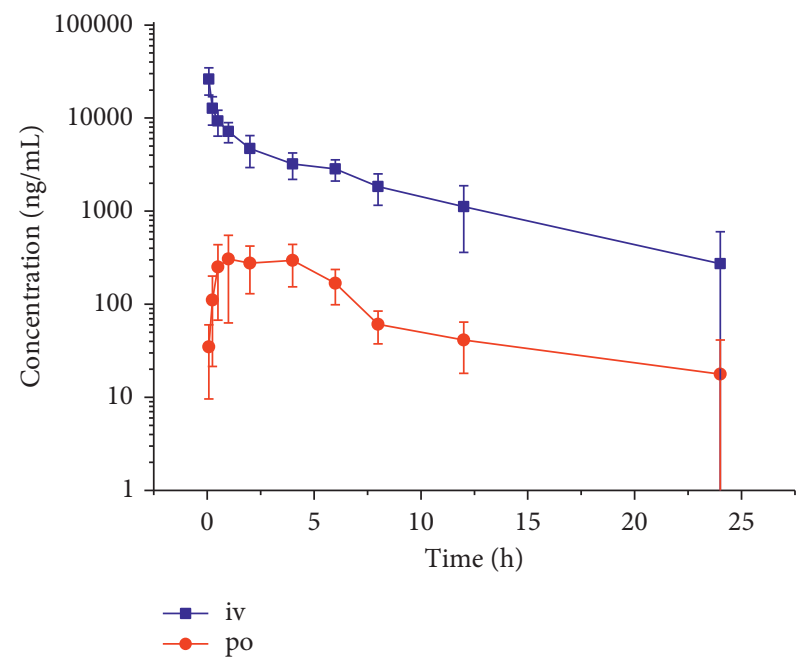

FIgURE 4: Mean blood concentration of TBMS-I after sublingual intravenous administration at the dose of $3 \mathrm{mg} / \mathrm{kg}$ and gavage of $15 \mathrm{mg} / \mathrm{kg}$.

TABLE 3: The pharmacokinetic parameters of TBMS-I after oral and intravenous administration $(n=6)$.

\begin{tabular}{lccc}
\hline Parameters & Unit & iv $(5 \mathrm{mg} / \mathrm{kg})$ & po $(20 \mathrm{mg} / \mathrm{kg})$ \\
\hline AUC $(0-t)$ & $\mathrm{ng} / \mathrm{mL} \cdot \mathrm{h}$ & $51205.8 \pm 13134.0$ & $2023.9 \pm 1145.5$ \\
AUC $(0-\infty)$ & $\mathrm{ng} / \mathrm{mL} \cdot \mathrm{h}$ & $59370.3 \pm 21468.0$ & $2051.8 \pm 1106.5$ \\
MRT $(0-t)$ & $\mathrm{h}$ & $5.1 \pm 1.8$ & $4.6 \pm 2.4$ \\
MRT $(0-\infty)$ & $\mathrm{h}$ & $8.9 \pm 7.0$ & $4.9 \pm 2.0$ \\
$t_{1 / 2 z}$ & $\mathrm{~h}$ & $6.8 \pm 5.6$ & $2.3 \pm 0.5$ \\
$\mathrm{CL}_{z / F}$ & $\mathrm{~L} / \mathrm{h} / \mathrm{kg}$ & 0.1 & $16.3 \pm 17.4$ \\
$T_{\max }$ & $\mathrm{H}$ & - & $1.8 \pm 1.3$ \\
$V_{z / F}$ & $\mathrm{~L} / \mathrm{kg}$ & 0.4 & $53.9 \pm 56.9$ \\
$C_{\max }$ & $\mathrm{ng} / \mathrm{mL}$ & $26192.5 \pm 8491.9$ & $429.6 \pm 164.9$ \\
\hline
\end{tabular}

determination of TBMS-I extracted from only $20 \mu \mathrm{L}$ of whole blood using a simple one-step protein precipitation procedure within $4 \mathrm{~min}$ was more sensitive, more convenient, and faster than traditional and commonly used analytical techniques. It is clear, in addition, that there are 
potentially large savings in the amount of compound and number of animals required for early pharmacokinetic studies. In the present study, the UPLC/MS/MS method has been successfully applied to the pharmacokinetic investigations of TBMS-I in mice after sublingual intravenous and intragastric administration. The oral bioavailability of tubeimoside I in mice is $1 \%$, which indicates that tubeimoside I is not easily absorbed into blood circulatory system through the gastrointestinal tract.

\section{Conflicts of Interest}

The authors declare that they have no conflicts of interest.

\section{Acknowledgments}

This research was supported by the Science and Technology Foundation of Wenzhou (Y20160540 and Y20150183) and a grant from the National Natural Science Foundation of China (81702388). The authors thank Wenzhou Medical University Analytical and Testing Center for excellent technical support.

\section{References}

[1] T. X. Yu, R. D. Ma, and L. J. Yu, "Structure-activity relationship of tubeimosides in anti-inflammatory, antitumor, and antitumor-promoting effects," Acta Pharmacologica Sinica, vol. 22, no. 5, pp. 463-468, 2001.

[2] Y. Gu, C. Korbel, C. Scheuer, A. Nenicu, M. D. Menger, and M. W. Laschke, "Tubeimoside-1 suppresses tumor angiogenesis by stimulation of proteasomal VEGFR2 and Tie2 degradation in a non-small cell lung cancer xenograft model," Oncotarget, vol. 7, no. 5, pp. 5258-5272, 2016.

[3] Q. Bian, P. Liu, J. Gu, and B. Song, "Tubeimoside-1 inhibits the growth and invasion of colorectal cancer cells through the Wnt/beta-catenin signaling pathway," International Journal of Clinical and Experimental Pathology, vol. 8, no. 10, pp. 12517-12524, 2015.

[4] G. Jia, Q. Wang, R. Wang et al., "Tubeimoside-1 induces glioma apoptosis through regulation of $\mathrm{Bax} / \mathrm{Bcl}-2$ and the ROS/Cytochrome C/Caspase-3 pathway," OncoTargets and Therapy, vol. 8, pp. 303-311, 2015.

[5] Y. Peng, Y. Zhong, and G. Li, "Tubeimoside-1 suppresses breast cancer metastasis through downregulation of CXCR4 chemokine receptor expression," BMB Reports, vol. 49, no. 9, pp. 502-507, 2016.

[6] M. J. Liang, W. D. Zhang, C. Zhang et al., "Quantitative determination of the anticancer agent tubeimoside $\mathrm{I}$ in rat plasma by liquid chromatography coupled with mass spectrometry," Journal of Chromatography B, vol. 845, no. 1, pp. 84-89, 2007.

[7] J. Kim, J. S. Min, D. Kim et al., "A simple and sensitive liquid chromatography-tandem mass spectrometry method for trans-epsilon-viniferin quantification in mouse plasma and its application to a pharmacokinetic study in mice," Journal of Pharmaceutical and Biomedical Analysis, vol. 134, pp. 116121, 2017.

[8] J. H. Xiong, H. Ye, Y. X. Lin et al., "Determining concentrations of icotinib in plasma of rat by UPLC method with ultraviolet detection: applications for pharmacokinetic studies," Current Pharmaceutical Analysis, vol. 13, no. 4, pp. 340-344, 2017.
[9] L. G. Chen, S. P. Yang, Z. Z. Liu et al., "Pharmacokinetic study of macitentan in rat plasma by ultra performance liquid chromatography-tandem mass spectrometry," Latin American Journal of Pharmacy, vol. 34, no. 7, pp. 1411-1416, 2015.

[10] L. G. Chen, Z. Wang, S. Wang, T. Li, Y. Pan, and X. Lai, "Determination of apremilast in rat plasma by UPLC-MS-MS and its application to a pharmacokinetic study," Journal of Chromatographic Science, vol. 54, no. 8, pp. 1336-1340, 2016.

[11] T. F. Yuan, S. T. Wang, and Y. Li, "Quantification of menadione from plasma and urine by a novel cysteaminederivatization based UPLC-MS/MS method," Journal of Chromatography B, vol. 1063, pp. 107-111, 2017.

[12] M. Sarkar, R. G. Grossman, E. G. Toups, and D. S. Chow, "UPLC-MS/MS assay of riluzole in human plasma and cerebrospinal fluid (CSF): application in samples from spinal cord injured patients," Journal of Pharmaceutical and Biomedical Analysis, vol. 146, pp. 334-340, 2017.

[13] M. Mueller, C. Maldonado-Adrian, J. Yuan, U. D. McCann, and G. A. Ricaurte, "Studies of (+/-)-3,4-methylenedioxymethamphetamine (MDMA) metabolism and disposition in rats and mice: relationship to neuroprotection and neurotoxicity profile," Journal of Pharmacology and Experimental Therapeutics, vol. 344, no. 2, pp. 479-488, 2013.

[14] K. Tatum-Gibbs, J. F. Wambaugh, K. P. Das et al., "Comparative pharmacokinetics of perfluorononanoic acid in rat and mouse," Toxicology, vol. 281, no. 1-3, pp. 48-55, 2011.

[15] P. L. Toutain, A. Ferran, and A. Bousquet-Melou, "Species differences in pharmacokinetics and pharmacodynamics," in Handbook of Experimental Pharmacology, pp. 19-48, Springer, Berlin, Germany, 2010.

[16] Y. Hu and D. E. Smith, "Species differences in the pharmacokinetics of cefadroxil as determined in wildtype and humanized PepT1 mice," Biochemical Pharmacology, vol. 107, pp. 81-90, 2016.

[17] Z. Li, C. Chen, D. Ai et al., "Pharmacokinetics and tissue residues of hydrochloric acid albendazole sulfoxide and its metabolites in crucian carp (Carassius auratus) after oral administration," Environmental Toxicology and Pharmacology, vol. 33, no. 2, pp. 197-204, 2012.

[18] S. M. Butler, M. A. Wallig, C. W. Nho et al., "A polyacetylenerich extract from Gymnaster koraiensis strongly inhibits colitis-associated colon cancer in mice," Food and Chemical Toxicology, vol. 53, pp. 235-239, 2013.

[19] K. C. Park, S. W. Kim, J. H. Park et al., "Potential anti-cancer activity of N-hydroxy-7-(2-naphthylthio) heptanomide (HNHA), a histone deacetylase inhibitor, against breast cancer both in vitro and in vivo," Cancer Science, vol. 102, no. 2, pp. 343-350, 2011.

[20] H. Yamazaki, H. Suemizu, M. Mitsui, M. Shimizu, and F. P. Guengerich, "Combining chimeric mice with humanized liver, mass spectrometry, and physiologically-based pharmacokinetic modeling in toxicology," Chemical Research in Toxicology, vol. 29, no. 12, pp. 1903-1911, 2016.

[21] V. Gota, J. S. Goda, K. Doshi et al., "Biodistribution and pharmacokinetic study of $3,3^{\prime}$ diseleno dipropionic acid (DSePA), a synthetic radioprotector, in mice," European Journal of Drug Metabolism and Pharmacokinetics, vol. 41, no. 6, pp. 839-844, 2016.

[22] R. Kumar, P. S. Suresh, G. Rudresh et al., "Determination of ulixertinib in mice plasma by LC-MS/MS and its application to a pharmacokinetic study in mice," Journal of Pharmaceutical and Biomedical Analysis, vol. 125, pp. 140-144, 2016.

[23] A. Watanabe, R. Watari, K. Ogawa et al., "Using improved serial blood sampling method of mice to study 
pharmacokinetics and drug-drug interaction," Journal of Pharmaceutical Sciences, vol. 104, no. 3, pp. 955-961, 2015.

[24] S. Gao, Z. Yang, T. Yin, M. You, and M. Hu, "Validated LC-MS/MS method for the determination of maackiain and its sulfate and glucuronide in blood: application to pharmacokinetic and disposition studies," Journal of Pharmaceutical and Biomedical Analysis, vol. 55, no. 2, pp. 288-293, 2011.

[25] US Department of Health and Human Services, Guidance for Industry: Bioanalytical Method Validation, F.a.D.A. US Department of Health and Human Services, Washington, DC, USA, 2013.

[26] W. Ye, R. Chen, W. Sun et al., "Determination and pharmacokinetics of engeletin in rat plasma by ultra-high performance liquid chromatography with tandem mass spectrometry," Journal of Chromatography B, vol. 1060, pp. 144-149, 2017.

[27] G. Y. Lin, L. F. Hu, X. Z. Yang, X. J. Pan, and X. Q. Wang, "Determination of gemcitabine in rabbit plasma by LC-ESIMS using an allure PFP propyl column," Latin American Journal of Pharmacy, vol. 30, no. 3, pp. 571-575, 2011.

[28] C. Ding, W. Dong, F. F. Yang et al., "Determination of ibudilast in rabbit plasma by liquid chromatography-mass spectrometry and its application," Latin American Journal of Pharmacy, vol. 30, no. 10, pp. 2065-2069, 2011.

[29] S. H. Wang, Z. X. Lin, K. Su et al., "Effect of curcumin and pirfenidone on toxicokinetics of paraquat in rat by UPLC-MS/ MS," Acta Chromatographica, vol. 30, no. 1, pp. 26-30, 2018.

[30] L. G. Chen, W. W. You, D. W. Chen et al., "Pharmacokinetic interaction study of ketamine and rhynchophylline in rat plasma by ultra-performance liquid chromatography tandem mass spectrometry," BioMed Research International, vol. 2018, Article ID 6562309, 8 pages, 2018.

[31] M. L. Zhang, J. Zhang, L. Y. Wan, X. L. Wu, C. C. Wen, and X. Q. Wang, "A simple and selective high performance liquid chromatography developed for determination diphenoxylate in rat plasma and tissues," Latin American Journal of Pharmacy, vol. 35, no. 10, pp. 2327-2330, 2016.

[32] J. Y. Guo, Q. Q. Xu, S. H. Tong et al., "The effect of transmetil on pharmacokinetics of MS-275 in rats," Latin American Journal of Pharmacy, vol. 33, no. 9, pp. 1567-1570, 2014.

[33] S. Wang, H. Wu, X. Huang et al., "Determination of $\mathrm{N}$-methylcytisine in rat plasma by UPLC-MS/MS and its application to pharmacokinetic study," Journal of Chromatography B, vol. 990, pp. 118-124, 2015.

[34] B. M. Fang, S. H. Bao, S. H. Wang et al., "Pharmacokinetic study of ardisiacrispin A in rat plasma after intravenous administration by UPLC-MS/MS," Biomedical Chromatography, vol. 31, no. 3, article e3826, 2017.

[35] S. Wang, H. Wu, P. Geng et al., "Pharmacokinetic study of dendrobine in rat plasma by ultra-performance liquid chromatography tandem mass spectrometry," Biomedical Chromatography, vol. 30, no. 7, pp. 1145-1149, 2016.

[36] E. Eliassen and L. Kristoffersen, "Quantitative determination of zopiclone and zolpidem in whole blood by liquid-liquid extraction and UHPLC-MS/MS," Journal of Chromatography B, vol. 971, pp. 72-80, 2014.

[37] M. K. Nielsen and S. S. Johansen, "Determination of olanzapine in whole blood using simple protein precipitation and liquid chromatography-tandem mass spectrometry," Journal of Analytical Toxicology, vol. 33, no. 4, pp. 212-217, 2009.

[38] C. C. Wen, S. H. Wang, X. L. Huang et al., "Determination and validation of hupehenine in rat plasma by UPLC-MS/MS and its application to pharmacokinetic study," Biomedical Chromatography, vol. 29, no. 12, pp. 1805-1810, 2015.
[39] C. Wen, Q. Zhang, Y. He, M. Deng, X. Wang, and J. Ma, "Gradient elution LC-MS determination of dasatinib in rat plasma and its pharmacokinetic study," Acta Chromatographica, vol. 27, no. 1, pp. 81-91, 2015.

[40] W. Q. Tian, J. Z. Cai, Y. Y. Xu et al., "Determination of xanthotoxin using a liquid chromatography-mass spectrometry and its application to pharmacokinetics and tissue distribution model in rat," International Journal of Clinical and Experimental Medicine, vol. 8, no. 9, pp. 15164-15172, 2015. 

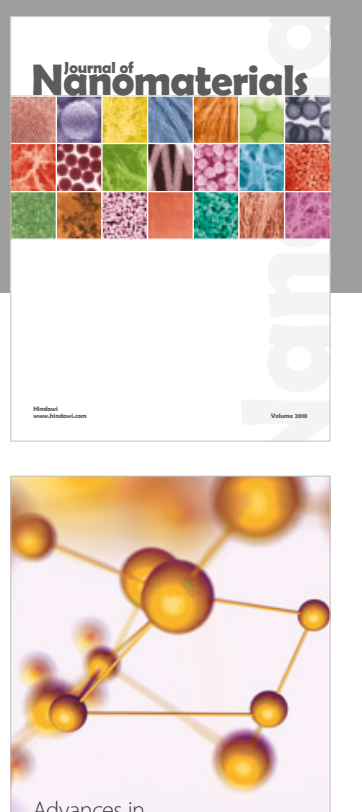

Physical Chemistry
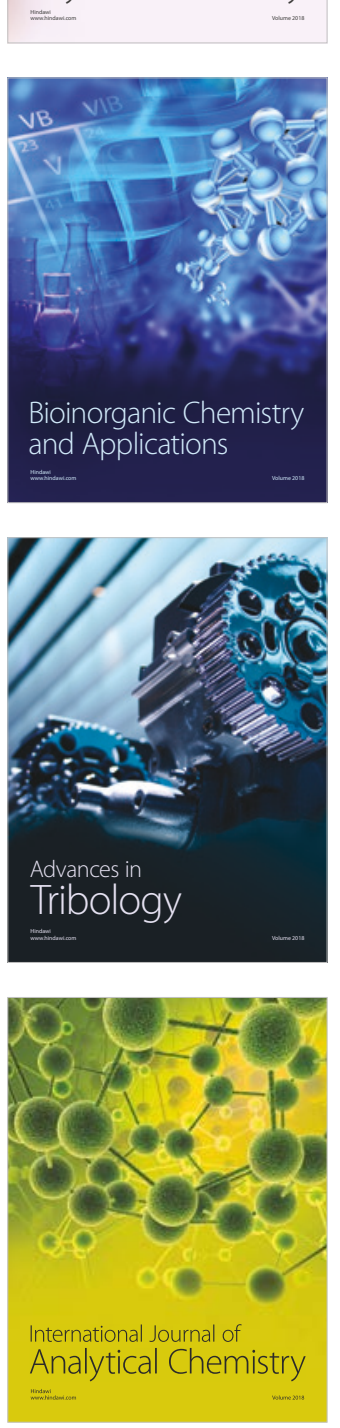

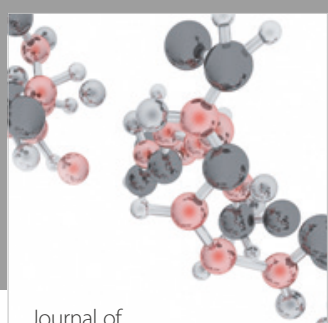

Analytical Methods

in Chemistry

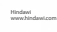

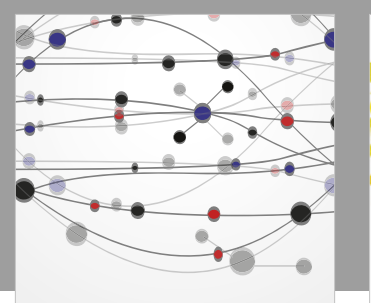

The Scientific World Journal

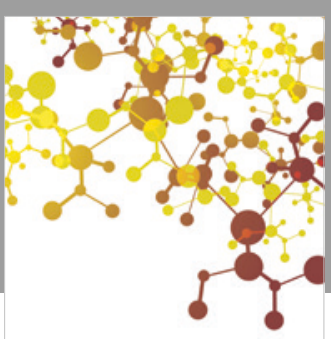

Journal of

Applied Chemistry
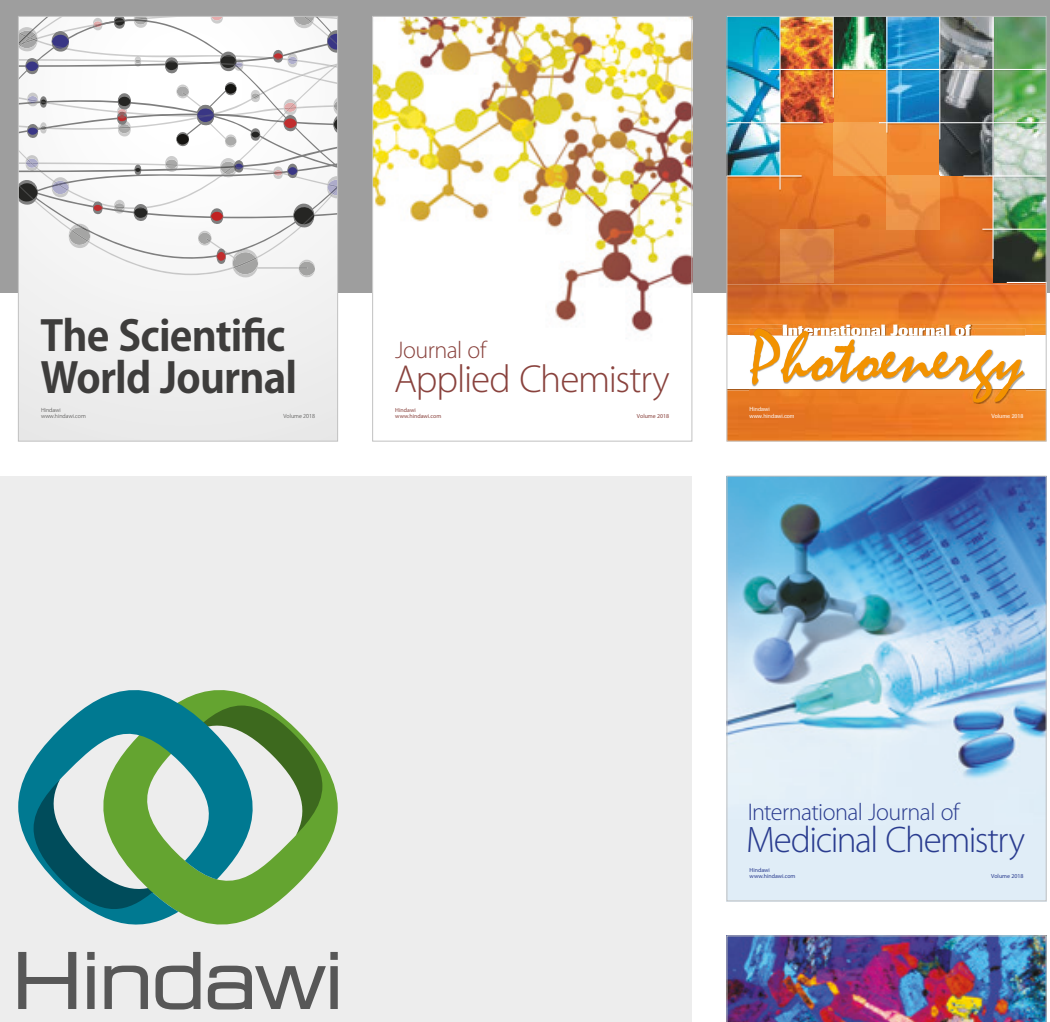

Submit your manuscripts at

www.hindawi.com
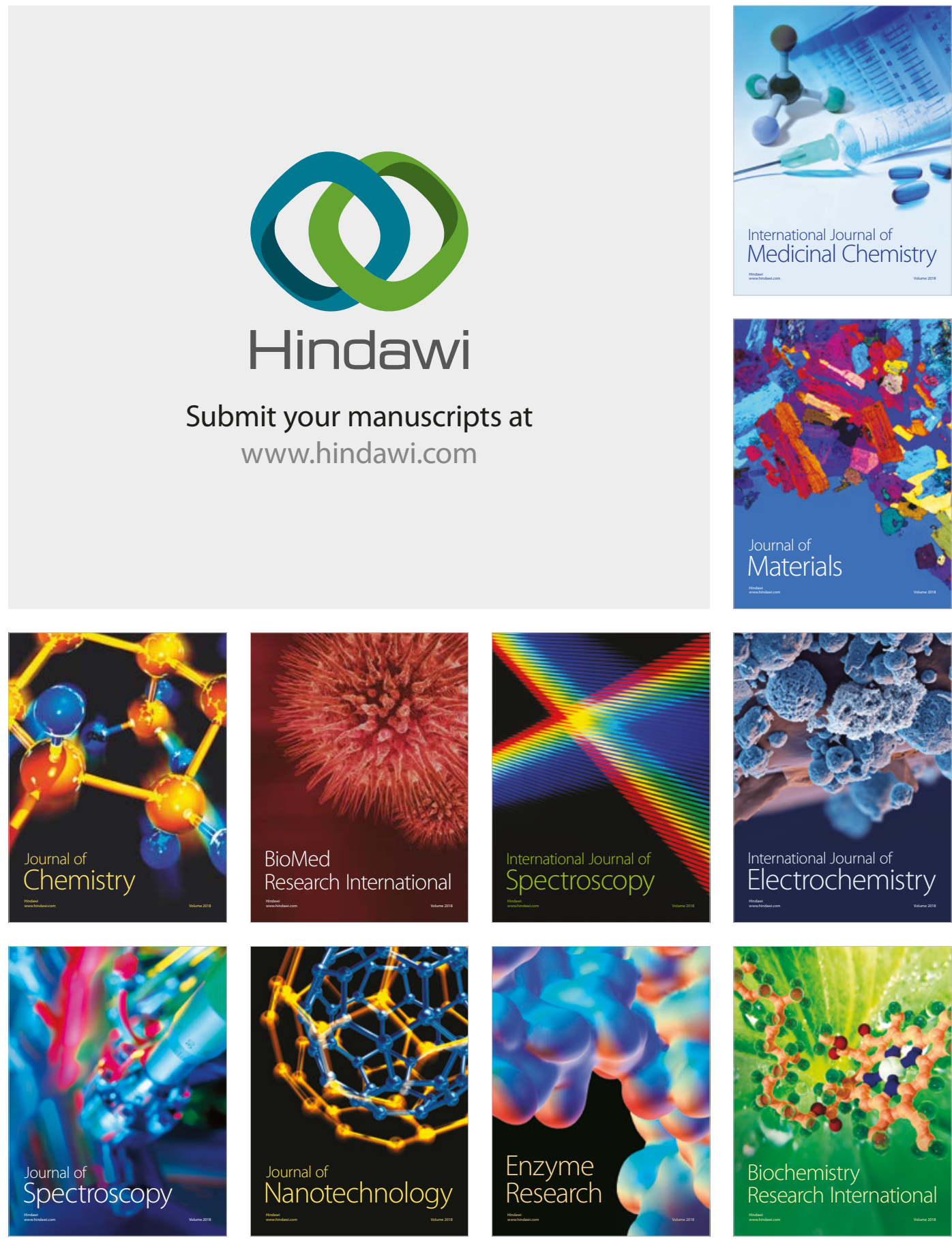
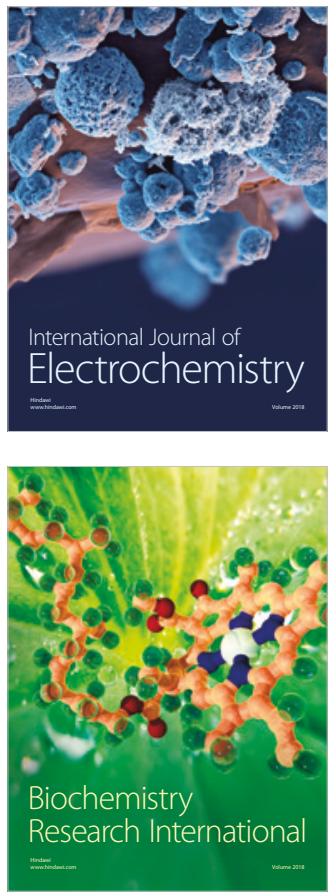